\title{
Complete Vascular Ring with Aortic Dissection in an Octogenarian
}

\author{
Satoshi Higuchi, Toshiyuki Takahashi, Yu Yamasaki, Yoko Shoji, Naoki Hirata, \\ Tasuku Hasegawa, Susumu Nakagawa and Hideo Mitamura
}

Key words: double aortic arch, complete vascular ring

(Inter Med 49: 1043, 2010)

(DOI: 10.2169/internalmedicine.49.3439)

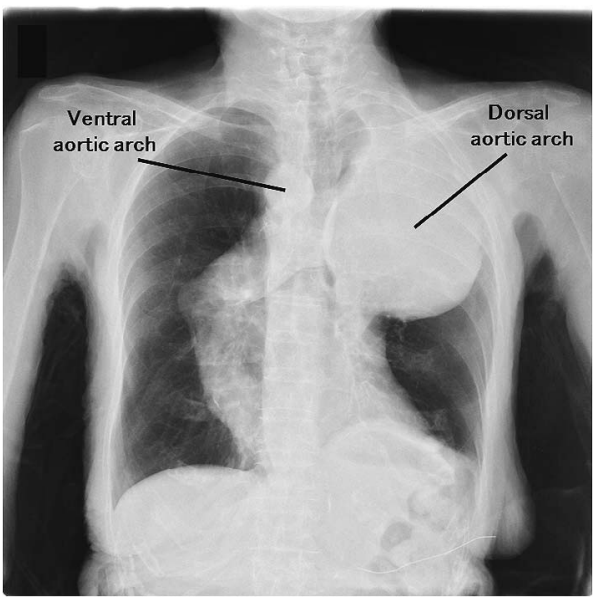

Picture 1. Chest X-ray shows a marked dilatation of the mediastinum with two abnormal circular shadows between which the trachea and the esophagus are located.

An 85-year-old woman presented with severe back pain. Chest X-ray showed a marked dilatation of the mediastinum with two abnormal circular shadows (Picture 1). Dynamic computed tomography (CT) revealed a double aortic arch with dissecting aneurysm in the left aortic arch, confirming the diagnosis of complete vascular ring (Picture 2). There were no stenoses of the trachea and the esophagus, which was uncommon in this anomalous condition. The patient re-

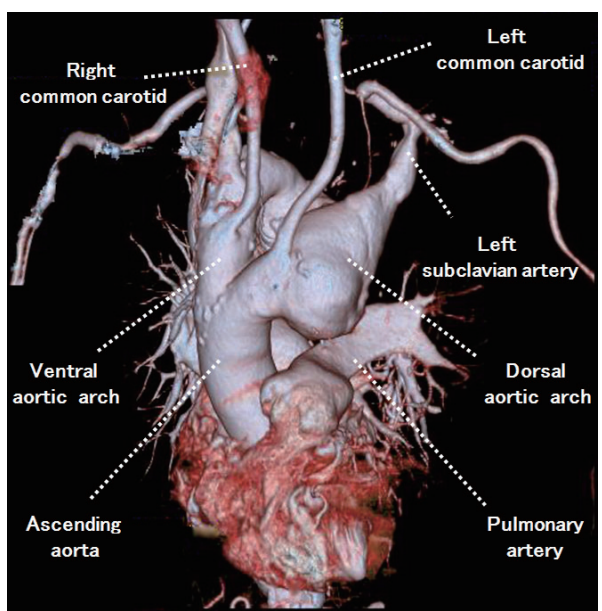

Picture 2. 3-dimensional CT reveals double aortic arch. The ascending aorta bifurcates into the dorsal and the ventral aorta which rejoin into a single descending aorta. The left common carotid and left subclavian arteries arise from the dorsal aortic arch, which forms a giant aneurysm. The right common carotid and right subclavian vessels arise from the ventral arch.

fused surgery and died from rupture of the aneurysm. In general, most patients with complete vascular ring have some symptoms related to tracheal and esophageal compression by the surrounding vascular ring, including dysphasia, vomiting, dyspnea and cough, by one year of age (1). This is a very rare case of complete vascular ring in an elderly woman who had been asymptomatic until the last episode of aortic dissection.

\section{Reference}

